\title{
Crystal Vibrations of Polyethylene
}

\author{
M. TASUMI* AND S. KRIMM \\ Harrison M. Randall Laboratory of Physics, University of Michigan, Ann Arbor, Michigan
}

(Received 18 August 1966)

\begin{abstract}
Several problems involving the internal and external vibrations of the polyethylene crystal have been studied. The splittings of some of the internal vibration bands arising from transition dipole coupling have been evaluated and found to have small but nonnegligible values as compared with the splittings calculated from the intermolecular $\mathrm{H} \cdots \mathrm{H}$ interaction potential. On the other hand, interactions between permanent $\mathrm{CH}_{2}$ dipole moments in different chains have been shown to make quite insignificant contributions to the translational lattice frequencies. The effects on the vibrational frequencies of cell contraction with decreasing temperature have been calculated, and the experimentally observed upward shift of a lattice frequency is found to be explainable primarily on this basis. The effect caused by the change of the setting angle of each chain in the unit cell has also been examined. The short-range $\mathrm{H} \cdot \mathrm{H} \mathrm{H}$ interaction force constants and the dispersion curves of normal and deuterated polyethylenes have been obtained.
\end{abstract}

\section{INTRODUCTION}

$I^{\mathrm{N}}$ $\mathrm{N}$ recent years there has been an increasing interest in the vibrational energy levels of crystalline polyethylene and $n$-paraffins. This has been particularly true for the low-frequency vibrations, which have been studied by means of neutron inelastic scattering, ${ }^{1-6}$ heatcapacity measurements, ${ }^{7-9}$ and spectroscopic methods. From the spectroscopic viewpoint, the establishment of the assignment of the $72-\mathrm{cm}^{-1}$ infrared band has been one of the most important problems. Bertie and Whalley ${ }^{10}$ have found that this band shifts to higher frequencies at low temperatures, and Frenzel and Butler ${ }^{11}$ have shown that this is a crystalline band. Krimm and Bank ${ }^{12}$ have reported that a corresponding band is observed in the spectrum of $n-\mathrm{C}_{100} \mathrm{D}_{202}$ at about $67 \mathrm{~cm}^{-1}$. They have also noted that the $72-\mathrm{cm}^{-1}$ band is not observed in triclinic $n-\mathrm{C}_{20} \mathrm{H}_{42}$. These experimental results strongly suggest the assignment of this band to the predicted spectroscopically active lattice mode. ${ }^{13}$ Calculations support this suggestion. Tasumi and Shimanouchi ${ }^{14}$ (hereafter cited as the TS

* Permanent address: Department of Chemistry, University of Tokyo, Bunkyo-ku, Tokyo, Japan.

1 H. R. Danner, G. J. Safford, H. Boutin, and M. Berger, J. Chem. Phys. 40, 1417 (1964).

${ }^{2}$ H. R. Danner, H. Boutin, and G. J. Safford, J. Chem. Phys. 41, 3649 (1964).

J. L. Donovan, Doctoral thesis, University of Michigan, Ann Arbor, Mich., 1964.

'W. Myers, J. L. Donovan, and J. S. King, J. Chem. Phys. 42,4299 (1965).

5 G. C. Summerfield, J. Chem. Phys. 43, 1079 (1965)

- W. Myers, Doctoral thesis, University of Michigan, Ann Arbor, Mich., 1965.

7 B. Wunderlich, J. Chem. Phys. 37, 1203 (1962).

${ }_{8} \mathrm{~T}$. Miyazawa and T. Kitagawa, Polymer Letters 2, 395 (1964).

${ }^{9} \mathrm{~T}$. Kitagawa and T. Miyazawa, Rept. Progr. Polymer Phys. Japan 8, 53 (1965)

${ }_{10}^{10}$ J. E. Bertie and E. Whalley, J. Chem. Phys. 41, 575 (1964). 11 A. O. Frenzel and J. P. Butler, J. Opt. Soc. Am. 54, 1059 (1964).

${ }_{12}$ S. Krimm and M. Bank, J. Chem. Phys. 42, 4059 (1965). ${ }^{13}$ S. Krimm, C. Y. Liang, and G. B. B. M. Sutherland, J. Chem. Phys. 25, 549 (1956)

${ }_{14}$ M. Tasumi and T. Shimanouchi, J. Chem. Phys. 43, 1245 (1965). calculation) have calculated the normal frequencies of the polyethylene crystal and determined the values of hydrogen-hydrogen interaction force constants. Through this calculation the factor group splittings of the $\mathrm{CH}_{2}$ bending and rocking vibrations have been basically explained, and the $72-\mathrm{cm}^{-1}$ band has been assigned to the $B_{1 u}$ translational lattice mode. This assignment has been supported by dichroism measurements recently done by Krimm and Bank. ${ }^{15}$ They have shown, using a sample having a special orientation, that this band is polarized along the $a$ axis of the crystal, as expected for the $B_{1 u}$ mode. A neutron-inelasticscattering experiment $t^{4}$ has shown several peaks which are consistent with some of the lattice frequencies predicted by the TS calculation. Thus, the interpretation of the vibrational spectrum of crystalline polyethylene have been successfully initiated.

Before a more detailed understanding of the spectrum can be obtained, several further problems need to be resolved. In this paper we wish to consider the following questions:

(1) To what extent does dipole-dipole interaction contribute to the lattice frequencies and to the magnitude of factor group splitting of internal vibrations? In the TS calculation this factor was assumed to be very small and was neglected. How valid is such an assumption?

(2) What effect does the setting angle $\theta$ (the angle between the $a$ axis of the crystal and the plane of the carbon skeleton; see Fig. 1) have on the frequencies in general? There is some disagreement concerning the value of this angle in several crystal structures determined by $\mathrm{x}$-ray diffraction. ${ }^{16-19}$ In the TS calculation a value of $42^{\circ}$ was used.

(3) What is the cause of the shift of the $72-\mathrm{cm}^{-1}$ band to higher frequencies as the temperature is

${ }^{15} \mathrm{~S}$. Krimm and M. Bank (to be published).

${ }^{16}$ C. W. Bunn, Trans, Faraday Soc. 35, 482 (1939).

17 A. E. Smith, J. Chem. Phys. 21, 2229 (1953).

18 H. M. Shearer and V. Vand, Acta Cryst. 9, 379 (1956).

${ }_{19}$ P. W. Teare, Acta Cryst. 12, 294 (1959). 


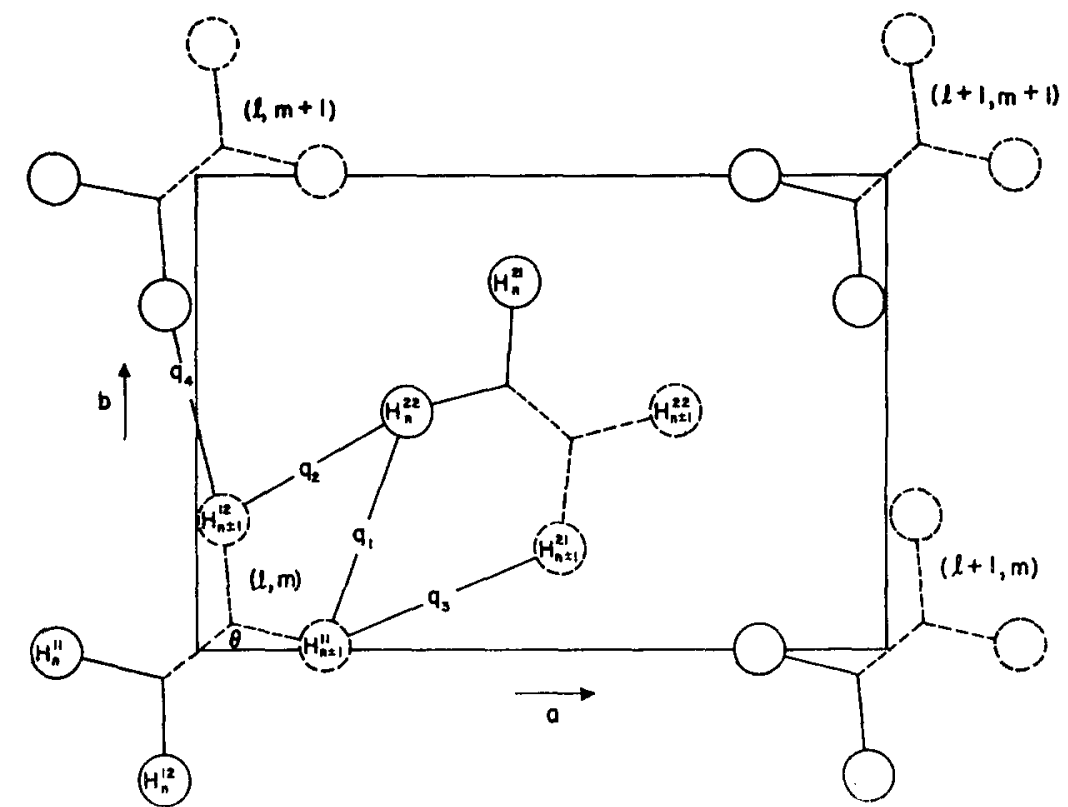

Fig. 1. Cross section of the unit cell of crystalline polyethylene. lowered? (The band shifts by about $6.5 \mathrm{~cm}^{-1}$ in going from $300^{\circ}$ to $\left.100^{\circ} \mathrm{K} .{ }^{10}\right)$ Krimm and $\mathrm{Bank}^{12}$ correlated this shift with the contraction in the cell dimensions with decreasing temperature. Their work, however, was based on a simplified model in which the polymer chain was approximated by a point mass. In this paper we calculate the effects of changes in cell dimensions using the complete structure.

\section{EFFECTS OF DIPOLE-DIPOLE INTERACTION ON INTERNAL AND LATTICE FREQUENCIES}

It has been shown that, for some organic molecular crystals, spectroscopic effects associated with intermolecular interactions can be explained satisfactorily by atom-atom interaction force constants..$^{14,20,21}$ However, in the case of polyethylene we do not know exactly the magnitude of splitting of the infrared bands which is expected from the interaction energy between the transition dipole moments in different chains. Nor do we know the contribution to the lattice frequencies of the interaction between the permanent $\mathrm{C}-\mathrm{H}$ moments or the $\mathrm{CH}_{2}$ group moments in different chains. Even for a crystal consisting of molecules having no total dipole moment, the bond or group moment might have some influence on the lattice frequencies. The effects of such interactions on internal and on lattice frequencies have been investigated and are discussed below.

\section{A. Splitting of the Infrared-Active $\mathrm{CH}_{2}$ Internal Vibrations}

Snyder ${ }^{22}$ has treated the effect of transition dipole interactions on the spectrum of polyethylene, and has

\footnotetext{
${ }^{20}$ D. A. Dows, Phys. Chem. Org. Solid State 1963, 657, and references cited there.

${ }^{21}$ I. Harada and T. Shimanouchi, J. Chem. Phys. 44, 2016 (1966).

${ }^{22}$ R. G. Snyder, J. Mol. Spectry. 7, 116 (1961).
}

found that rather unreasonable values have to be assumed for the permanent $\mathrm{C}-\mathrm{H}$ bond moment and its derivative with respect to a bending coordinate if the splittings of the $\mathrm{CH}_{2}$ rocking-twisting bands are to be accounted for. [The observed frequencies of the infrared-active $\mathrm{CH}_{2}$ bending modes are $1473\left(B_{1 u}\right)$ and $1463\left(B_{2 u}\right) \mathrm{cm}^{-1}\left(10-\mathrm{cm}^{-1}\right.$ splitting), and those of the $\mathrm{CH}_{2}$ rocking modes are $731\left(B_{1 u}\right)$ and $720\left(B_{2 u}\right)$ (11- $\mathrm{cm}^{-1}$ splitting) at room temperature. $\left.{ }^{13}\right]$ This has suggested that transition dipole coupling may not be significant in contributing to the observed splittings. However, it is also possible that the bond-moment approach is not a satisfactory one. The interaction of group transition dipoles might be expected to be a better starting point for the calculation of such effects. We have made an estimate of the magnitude of such transition dipole coupling, utilizing the group-moment derivatives obtained by Snyder. ${ }^{23}$ The group-moment derivative is the quantity represented by $\partial \boldsymbol{u} / \partial S$, where $\boldsymbol{u}$ is the molecular dipole moment and $S$ a group symmetry coordinate. The group-moment derivatives given for the $\mathrm{CH}_{2}$ bending and rocking coordinates are, respectively, 0.30 and $0.33 \mathrm{D} / \mathrm{rad}$. Those of the $\mathrm{CH}_{2}$ symmetric and antisymmetric stretching coordinates are 0.7 and $0.98 \mathrm{D} / \AA$. The interaction energy $\left(V_{d}\right)$ between two transition dipoles may be written, as usual,

$$
\begin{aligned}
V_{d} & =\left(\left|\boldsymbol{u}_{1}\right|\left|\mathbf{u}_{2}\right| /|\mathbf{R}|^{3}\right)(\cos \alpha-3 \cos \beta \cos \gamma) \\
& =\frac{\left|\partial \mathbf{u} / \partial S_{1}\right|\left|\partial \mathbf{u} / \partial S_{2}\right|}{|\mathbf{R}|^{3}}(\cos \alpha-3 \cos \beta \cos \gamma) S_{1} S_{2},
\end{aligned}
$$

where $\mathbf{R}$ is the vector connecting the centers of two dipoles, and $\alpha, \beta, \gamma$ are defined as

$$
\begin{gathered}
\left|\boldsymbol{u}_{1}\right|\left|\boldsymbol{u}_{2}\right| \cos \alpha=\boldsymbol{u}_{1} \cdot \boldsymbol{u}_{2}, \quad\left|\boldsymbol{u}_{1}\right||\mathbf{R}| \cos \beta=\boldsymbol{u}_{1} \cdot \mathbf{R}, \\
\left|\boldsymbol{u}_{2}\right||\mathbf{R}| \cos \gamma=\boldsymbol{u}_{2} \cdot \mathbf{R} .
\end{gathered}
$$

${ }^{23}$ R. G. Snyder, J. Chem. Phys. 42, 1744 (1965). 
TABLE I. Frequency shifts and splittings due to dipole-dipole interactions.

\begin{tabular}{|c|c|c|c|c|c|c|c|c|c|}
\hline \multirow{3}{*}{ Mode } & \multirow{3}{*}{$\begin{array}{l}\text { Crystal } \\
\text { symmetry }\end{array}$} & \multicolumn{4}{|c|}{ Frequency shift from single chain ${ }^{a}\left(\mathrm{~cm}^{-1}\right)$} & \multicolumn{4}{|c|}{ Splitting, $\bar{\nu}\left(B_{1 u}\right)-\bar{\nu}\left(B_{2 u}\right)\left(\mathrm{cm}^{-1}\right)$} \\
\hline & & \multicolumn{2}{|c|}{$A$} & \multicolumn{2}{|c|}{$B$} & \multicolumn{2}{|c|}{$A$} & \multicolumn{2}{|c|}{$B$} \\
\hline & & $6 \AA$ & $9 \AA$ & $6 \AA$ & $9 \AA$ & $6 \AA$ & $9 \AA$ & $6 \AA$ & $9 \AA$ \\
\hline $\mathrm{CH}_{2}$ bending & $\begin{array}{l}B_{1 u} \\
B_{2 u}\end{array}$ & $\begin{array}{l}-1.0 \\
+1.2\end{array}$ & $\begin{array}{l}-0.9 \\
+0.9\end{array}$ & $\begin{array}{l}-1.2 \\
+1.1\end{array}$ & $\begin{array}{l}-1.2 \\
+0.7\end{array}$ & -2.2 & -1.8 & -2.3 & -1.9 \\
\hline $\mathrm{CH}_{2}$ rocking & $\begin{array}{l}B_{1 u} \\
B_{2 u}\end{array}$ & $\begin{array}{l}-1.0 \\
+0.2\end{array}$ & $\begin{array}{l}-1.0 \\
+0.1\end{array}$ & $\begin{array}{r}-0.9 \\
+0.3\end{array}$ & $\begin{array}{l}-0.9 \\
+0.2\end{array}$ & -1.2 & -1.1 & -1.2 & -1.1 \\
\hline $\mathrm{CH}_{2}$ sym stretch & $\begin{array}{l}B_{\mathrm{I} u} \\
B_{2 u}\end{array}$ & $\begin{array}{r}-1.2 \\
+1.4\end{array}$ & $\begin{array}{l}-1.2 \\
+1.2\end{array}$ & $\begin{array}{l}-1.6 \\
+1.3\end{array}$ & $\begin{array}{l}-1.6 \\
+1.1\end{array}$ & -2.6 & -2.4 & -2.9 & -2.7 \\
\hline $\mathrm{CH}_{2}$ antisym stretch & $\begin{array}{l}B_{1 u} \\
B_{2 u}\end{array}$ & $\begin{array}{l}-4.7 \\
+1.6\end{array}$ & $\begin{array}{l}-4.8 \\
+0.8\end{array}$ & $\begin{array}{l}-4.2 \\
+2.2\end{array}$ & $\begin{array}{l}-4.4 \\
+1.6\end{array}$ & -6.3 & -5.6 & -6.4 & -6.0 \\
\hline
\end{tabular}

Frequencies calculated for a single chain are $1461.6,700.0,2846.2$, and $2908.3 \mathrm{~cm}^{-1}$ for $\mathrm{CH}_{2}$ bending, rocking, symmetric stretching, and antisymmetric stretching modes, respectively.

The location within a group of the center of a transition moment is difficult to know. The interaction energy was calculated therefore for two such positions: one is the intersection between the bisector of the $\mathrm{HCH}$ angle and the line connecting the two hydrogen atoms of the $\mathrm{CH}_{2}$ group (A position), and the other is halfway between the $A$ position and the carbon atom ( $B$ position). The number of pairs of interacting dipoles which should be considered is also a problem. We calculated the splitting first by taking 30 pairs of interactions within a sphere of about $6-\AA$ radius from any one $\mathrm{CH}_{2}$ group, and then by taking 106 pairs of interactions within about a $9-\AA$ radius. Only interactions with $\mathrm{CH}_{2}$ group dipoles in other chains were considered. The directions of the dipole derivatives of both the $\mathrm{CH}_{2}$ bending and the symmetric stretching coordinates coincide with the bisector of the $\mathrm{HCH}$ angle, and those of the $\mathrm{CH}_{2}$ rocking and antisymmetric stretching coordinates are parallel to the line connecting the two hydrogen atoms. The setting angle $\theta$ was assumed to be $48^{\circ}$. The molecular geometry that was used is nontetrahedral, and is described in Sec. III. Using this geometry the values of the interaction energies may be calculated. Treating the energy as a perturbation to the potential energy of a single chain, we can evaluate the single-chain frequency shifts and the splittings between the $B_{1 u}$ and $B_{2 u}$ frequencies. ${ }^{14}$ The results are given in Table $I$ for room temperature unit cell parameters. It should be noted that (1) the values of the shifts and splittings calculated from the dipole interactions within the $9-\AA$ radius are in general only slightly smaller than those from the $6-\AA$ radius calculation, and (2) the change in position of the dipole center from the $\mathbf{A}$ to the $\mathbf{B}$ position makes little difference in the results of the calculation. Since the interaction energy is proportional to $\mathbf{R}^{-3}$, we expect dipole pairs farther apart than $9 \AA$ to make no significant contribution to the splitting. We may therefore regard the values for the $9-\AA$ radius as probably the upper limits to the shifts and splittings arising from transition dipole coupling. The splittings calculated for the $\mathrm{CH}_{2}$ bending and rocking modes are both small compared with the observed values. Of particular interest, however, is the fact that the $B_{2 u}$ frequencies of both of these modes are calculated to be higher than the $B_{1 u}$ frequencies. This is opposite to the experimental findings, and implies that the atom-atom interaction energy dominates the dipole-dipole interaction energy to produce the observed splittings of +10 and $+11 \mathrm{~cm}^{-1}$ for the $\mathrm{CH}_{2}$ bending and rocking modes. For the $\mathrm{CH}_{2}$ symmetric and antisymmetric stretching modes the splittings calculated from the atom-atom interaction potential gave values of -3.0 and $+2.3 \mathrm{~cm}^{-1}$, 24 thus predicting a total splitting of -5.4 and $-3.3 \mathrm{~cm}^{-1}$, respectively (taking the dipole splitting calculated for the $9-\AA$ radius and the A position). The result for the symmetric stretching vibration is consistent with experimental findings for $n$ - $\mathrm{C}_{36} \mathrm{H}_{74} \cdot{ }^{13}$ Using polarized radiation, the $\mathrm{CH}_{2}$ symmetric stretching band showed a doublet at 2857 and $2850 \mathrm{~cm}^{-1}$, with these components polarized along the $b$ and $a$ axes, respectively. This shows that the $B_{2 u}$ frequency is higher than the $B_{1 u}$ frequency, and thus the observed splitting is $-7 \mathrm{~cm}^{-1}$. No clear splitting was observed for the $\mathrm{CH}_{2}$ antisymmetric stretching band at about $2900 \mathrm{~cm}^{-1}$ because of its high intensity.

\section{B. Translational Lattice Vibrations}

For simplicity we assume that the chains move rigidly in the translational lattice vibrations, although actually very small coupling may take place between the lattice and internal vibrations. ${ }^{14}$ The chains move

\footnotetext{
${ }^{24}$ These values are quoted from Ref. 14 . In our new calculation based on the Set II force constants these values are +0.8 and $-1.9 \mathrm{~cm}^{-1}$ giving predicted total splittings of -1.6 and $-7.5 \mathrm{~cm}^{-1}$.
} 
parallel to the a axis in the $B_{2 u}$ mode and parallel to the $b$ axis in the $B_{1 u}$ mode.

Let $\lambda_{T i}$ be the frequency parameter of a translational lattice vibration $\left(\lambda_{T i}=4 \pi^{2} c^{2} \bar{\nu}_{T i}^{2} ; \bar{\nu}_{T i}\right.$ is the lattice vibration frequency in wavenumbers). We divide this into two parts, $\lambda_{T_{i}{ }^{a}}$ and $\lambda_{T i}{ }^{d}$. These two represent, respectively, the contribution from the atom-atom potential and that from the dipole-dipole potential. Then $\lambda_{T i}{ }^{d}$ may be written as

$$
\lambda_{T i}{ }^{d}=\partial^{2} V_{d} / \partial Q_{T_{i}{ }^{2}} \quad(i=a, b),
$$

where $Q_{T i}$ is the normal coordinate of a translational vibration $\left(Q_{T a}\right.$ and $Q_{T b}$ represent the $B_{2 u}$ and $B_{1 u}$ modes, respectively). $Q_{T^{\prime} i}$ may be expressed in terms of the displacement coordinate $\left(\Delta G_{i}\right)$ of the center of gravity of the $\mathrm{CH}_{2}$ group as,

$$
Q_{T i}=(m / 2 N)^{1 / 2}\left(\sum_{n} \Delta G_{i 1 n}-\sum_{n^{\prime}} \Delta G_{i 2 n^{\prime}}\right),
$$

where $\Delta G_{i n n}$ and $\Delta G_{i 2 n^{\prime}}$ refer to Chains 1 and $2, m$ is the mass of the $\mathrm{CH}_{2}$ group, and $\mathrm{N}$ the number of $\mathrm{CH}_{2}$ groups in a chain. Letting $V_{d}=\sum_{n} \sum_{n^{\prime}} V_{d}\left(\begin{array}{l}1 n \\ 2 n^{\prime}\end{array}\right)$, where $V_{d}\left(\begin{array}{c}1 n \\ 2 n^{\prime}\end{array}\right)$ is the interaction energy between the methylene groups $1 n$ and $2 n^{\prime}$, and using (4), we obtain

$$
\begin{aligned}
\partial^{2} V_{d} / \partial Q_{r_{i}{ }^{2}} & =(2 N m)^{-1} \\
& \times \sum_{n} \sum_{n^{\prime}}\left\{\left[\partial^{2} V_{d}\left(\begin{array}{c}
1 n \\
2 n^{\prime}
\end{array}\right) / \partial \Delta G_{i 1 n^{2}}{ }^{2}\right]-2\left[\partial^{2} V_{d}\left(\begin{array}{c}
1 n \\
2 n^{\prime}
\end{array}\right) / \partial \Delta G_{i 1 n^{2}} \partial \Delta G_{i 2 n^{\prime}}\right]+\left[\partial^{2} V_{d}\left(\begin{array}{c}
1 n \\
2 n^{\prime}
\end{array}\right) / \partial \Delta G_{i 2 n^{\prime}}{ }^{2}\right]\right\} .
\end{aligned}
$$

The calculation of the second derivatives for various pairs of interacting dipoles is a rather lengthy process. We have assumed a permanent $\mathrm{CH}_{2}$ group moment $\mu$ (not $\mathrm{CH}$ bond moments) and taken into account the interaction between the $n$th $\mathrm{CH}_{2}$ group in a central chain and the $n$ th, $(n \pm 1)$ th and the $(n \pm 2)$ th $\mathrm{CH}_{2}$ groups in the four nearest surrounding chains of different orientation. The results are [in (debye) ${ }^{2}$ (ang-

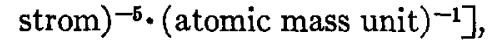

$$
\begin{aligned}
& \lambda_{T a}{ }^{d}=\partial^{2} V_{d} / \partial Q_{T a^{2}}{ }^{2}=-0.090\left(\mu^{2} / m\right), \\
& \lambda_{T b^{d}}{ }^{2}=\partial^{2} V_{d} / \partial Q_{T b}{ }^{2}=+0.094\left(\mu^{2} / m\right) .
\end{aligned}
$$

Both $\lambda_{T a}{ }^{d}$ and $\lambda_{T b}{ }^{d}$ are apparently very small. Even if we assume $\mu$ to be as large as $0.7 \mathrm{D}$, the $B_{2 u}$ frequency, which was calculated to be about $110 \mathrm{~cm}^{-1}$ from the atom-atom potential, ${ }^{14}$ would shift downward by only about $2.5 \mathrm{~cm}^{-1}$, and the $B_{1 u}$ frequency located at $72 \mathrm{~cm}^{-1}$ would shift upward by about $4 \mathrm{~cm}^{-1}$. The inclusion of longer range interaction distances is not likely to change this result significantly.

These results suggest that the atom-atom potential plays the major role in determining the lattice frequencies in polyethylene, the dipole-dipole coupling contributing no more than the order of a couple percent (probably much less) to the frequencies. On the other hand, while the atom-atom potential is dominant in accounting for the splittings of the $\mathrm{CH}_{2}$ rocking and bending modes, transition dipole coupling is not a negligible factor, contributing of the order of $10 \%$ in the former case and $20 \%$ in the latter case to the frequency splitting. For the $\mathrm{CH}_{2}$ stretching modes it appears that the relative contribution of transition dipole coupling to the splitting is even larger, probably because of the intrinsically larger transition moments for these modes. The polyethylene crystal may thus provide an optimum system in which to study the relative magnitudes of nonbonded interactions and transition dipole interactions between molecules.

\section{DYNAMICAL MATRIX}

In this section we discuss some details of setting up the calculation for the lattice modes of polyethylene. On the basis of the results from the previous section, only the effect of the atom-atom potential is considered.

We label a unit cell with indices $\left(l, m, n^{0}\right)$ where $l, m$, and $n^{0}$ run, respectively, along the $a, b$, and $c$ crystallographic axes. In the TS calculation $n$ was used for labeling the $\mathrm{CH}_{2}$ groups along the $\mathrm{C}_{2} \mathrm{~S}(c)$ axis. Here we use $n^{0}$ to label unit cells in the direction of the $c$ axis. We define the $\left(n^{0}+i\right)$ th cell such that it has the $(n+2 i)$ th and $(n+2 i-1)$ th $\mathrm{CH}_{2}$ groups in it [for example, the $n^{0}$ th cell is made up of the $n$th and $(n-1)$ th $\mathrm{CH}_{2}$ groups]. We next introduce the phase differences between corresponding motions in unit cells along the three crystallographic axes, namely, $\phi_{a}, \phi_{b}$, and $\phi_{c}$. In the TS calculation the phase difference $\left(\delta_{c}\right)$ between adjacent $\mathrm{CH}_{2}$ groups along the $c$ axis was used. The relationship between $\delta_{c}$ and $\phi_{c}$ is simply written as $2 \delta_{c}=\phi_{c}$. The vibrations in the unit cell $\left(l, m, n^{0}\right)$ have the phase angle $\left(l \phi_{a}+m \phi_{b}+n^{0} \phi_{c}\right)$.

Following the TS calculation, we assume four $\mathrm{H} \cdots \mathrm{H}$ force constants, $f_{1}, f_{2}, f_{3}$ and $f_{4}$, for the $\mathrm{H} \cdots \mathrm{H}$ distances, $q_{1}, q_{2}, q_{3}$ and $q_{4}$ (see Fig. 1). Since we are taking such short-range force constants, the intermolecular potential energy matrix $\mathbf{F}^{\prime}$ pertinent to the cell $\left(l, m, n^{0}\right)$ consists of a relatively small number of interactions. $\mathrm{F}^{\prime}$ may be expressed in terms of the $a-b$-, and $c$ displacement coordinates of each atom. We represent the interchain interaction within $\left(l, m, n^{0}\right)$ by the 
submatrix $\mathrm{F}^{\prime}\left(l, m, n^{0} ; l, m, n^{0}\right)$, the interaction between $\left(l, m, n^{0}\right)$ and $\left(l, m, n^{0} \pm 1\right)$ by $\mathbf{F}^{\prime}\left(l, m, n^{0} ; l, m, n^{0} \pm 1\right)$, and so forth. The order of such a submatrix is equal to the number of vibrational degrees of freedom of the atoms within a cell, i.e., 36 in this case. However, the elements pertinent to the coordinates of the carbon atoms are all equal to zero, reflecting the fact that no force constants are assumed for the atomic pairs C...C and C $\cdots H$. (This would be expected to be a satisfactory assumption for polyethylene. ${ }^{21}$ ) The matrix $\mathrm{F}^{\prime}\left(\phi_{a}, \phi_{b}, \phi_{c}\right)$, which is associated with vibrations having the phase differences $\phi_{a}, \phi_{b}$, and $\phi_{c}$, may be obtained by multiplying the submatrices with the following phase factors:

$$
\begin{aligned}
\mathrm{F}^{\prime}\left(\phi_{a}, \phi_{b}, \phi_{c}\right)= & \mathrm{F}^{\prime}\left(l, m, n^{0} ; l, m, n^{0}\right) \\
& +\mathrm{F}^{\prime}\left(l, m, n^{0} ; l, m, n^{0} \pm 1\right) \exp \left( \pm i \phi_{c}\right) \\
& +\mathrm{F}^{\prime}\left(l, m, n^{0} ; l \pm 1, m, n^{0}\right) \exp \left( \pm i \phi_{a}\right) \\
& +\mathrm{F}^{\prime}\left(l, m, n^{0} ; l+1, m, n^{0} \pm 1\right) \exp \left(+i \phi_{a} \pm i \phi_{c}\right) \\
& +\mathrm{F}^{\prime}\left(l, m, n^{0} ; l-1, m, n^{0} \pm 1\right) \exp \left(-i \phi_{a} \pm i \phi_{c}\right) \\
& +\mathrm{F}^{\prime}\left(l, m, n^{0} ; l, m \pm 1, n^{0}\right) \exp \left( \pm i \phi_{b}\right) \\
& +\mathrm{F}^{\prime}\left(l, m, n^{0} ; l, m+1, n^{0} \pm 1\right) \exp \left(+i \phi_{b} \pm i \phi_{c}\right) \\
& +\mathrm{F}^{\prime}\left(l, m, n^{0} ; l, m-1, n^{0} \pm 1\right) \exp \left(-i \phi_{b} \pm i \phi_{c}\right) \\
& +\mathrm{F}^{\prime}\left(l, m, n^{0} ; l \pm 1, m \pm 1, n^{0}\right) \exp \left( \pm i \phi_{a} \pm i \phi_{b}\right) \\
& +\mathrm{F}^{\prime}\left(l, m, n^{0} ; l+1, m+1, n^{0} \pm 1\right) \exp \left(+i \phi_{a}+i \phi_{b} \pm i \phi_{c}\right) \\
& +\mathrm{F}^{\prime}\left(l, m, n^{0} ; l-1, m-1, n^{0} \pm 1\right) \exp \left(-i \phi_{a}-i \phi_{b} \pm i \phi_{c}\right) .
\end{aligned}
$$

$\mathbf{F}^{\prime}\left(\phi_{a}, \phi_{b}, \phi_{c}\right)$ is a Hermitian matrix. However, we have found that we may reduce it to a real and symmetric

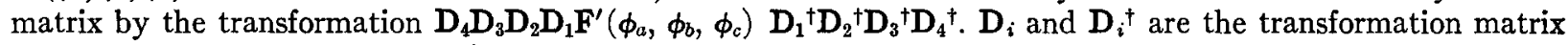
and its complex conjugate $\left(\mathbf{D}_{i} \mathbf{D}_{i}{ }^{\dagger}=\mathbf{E}\right)$.

(1) $D_{1}: D_{1}$ is a diagonal matrix and the diagonal elements are:

$$
\begin{array}{ll}
\exp \left(-i \frac{3}{4} \phi_{a}-i \frac{1}{4} \phi_{b}\right) & \text { for the } n \text {th } \mathrm{H}_{11} \text { coordinates, } \\
\exp \left(+i \frac{1}{4} \phi_{a}-i \frac{1}{4} \phi_{b}-i \frac{1}{2} \phi_{c}\right) & \text { for the }(n-1) \text { th } \mathrm{H}_{11} \text { coordinates, } \\
\exp \left(-i \frac{1}{4} \phi_{a}-i \frac{3}{4} \phi_{b}\right) & \text { for the } n \text {th } \mathrm{H}_{12} \text { coordinates, } \\
\exp \left(-i \frac{1}{4} \phi_{a}+i \frac{1}{4} \phi_{b}-i \frac{1}{2} \phi_{c}\right) & \text { for the }(n-1) \text { th } \mathrm{H}_{12} \text { coordinates, } \\
\exp \left(+i \frac{1}{4} \phi_{a}+i \frac{3}{4} \phi_{b}\right) & \text { for the } n \text {th } \mathrm{H}_{21} \text { coordinates, } \\
\exp \left(+i \frac{1}{4} \phi_{a}-i \frac{1}{4} \phi_{b}-i \frac{1}{2} \phi_{c}\right) & \text { for the }(n-1) \text { th } \mathrm{H}_{21} \text { coordinates, } \\
\exp \left(-i \frac{1}{4} \phi_{a}+i \frac{1}{4} \phi_{b}\right) & \text { for the } n \text {th } \mathrm{H}_{22} \text { coordinates, } \\
\exp \left(+i \frac{3}{4} \phi_{a}+i \frac{1}{4} \phi_{b}-i \frac{1}{2} \phi_{c}\right) & \text { for the }(n-1) \text { th } \mathrm{H}_{22} \text { coordinates. }
\end{array}
$$

(2) $D_{2}: D_{2}$ is also a diagonal matrix and the diagonal elements are:

1 for the $a$ and $b$ coordinates of both the $n$th and $(n-1)$ th $\mathrm{H}$ atoms,

$-i$ for the $c$ coordinates of the $n$th $\mathrm{H}$ atoms,

$i \quad$ for the $c$ coordinates of the $(n-1)$ th $\mathrm{H}$ atoms.

(3) $\mathrm{D}_{3}$ : By this block diagonal transformation matrix we set up the symmetric and antisymmetric combinations of the $n$th and $(n-1)$ th coordinates of any kind of $\mathrm{H}$ atoms $\left(\mathrm{H}_{11}, \mathrm{H}_{12}, \mathrm{H}_{21}\right.$, and $\left.\mathrm{H}_{22}\right)$ :

$$
\left(\begin{array}{c}
a+ \\
a-
\end{array}\right)=\left(\begin{array}{cc}
2^{-1 / 2} & 2^{-1 / 2} \\
2^{-1 / 2} & -2^{-1 / 2}
\end{array}\right)\left(\begin{array}{c}
n \text {th } a \text { coordinate } \\
(n-1) \text { th } a \text { coordinate }
\end{array}\right),
$$

where the first matrix on the right-hand side of (11) is one of the blocks of $\mathrm{D}_{3}$. The $b+, b-, c+, c-$ coordinates may be defined similarly. 
(4) $\mathrm{D}_{4}$ : This is again a diagonal matrix and the diagonal elements are:

1 for the symmetric coordinates $(a+, b+$, and $c+)$,

$-i$ for the antisymmetric coordinates $(a-, b-$, and $c-)$.

Such transformations do not reduce the order of the matrix, but they can reduce the number of nonzero off-diagonal elements greatly. After the above transformations, $\mathbf{F}^{\prime}\left(\phi_{a}, \phi_{b}, \phi_{c}\right)$ is factored essentially into symmetric $(+)$ and antisymmetric ( - ) parts, and only the cross terms between the symmetric $\mathrm{H}_{11}$ and antisymmetric $\mathrm{H}_{22}$ coordinates and those between the antisymmetric $\mathrm{H}_{11}$ and symmetric $\mathrm{H}_{22}$ coordinates do not vanish. This matrix can be rigorously factored, however, when $\phi_{a}=\phi_{b}=0$, and in this special case it can be reduced to a matrix of the ninth order. ${ }^{14}$

The intramolecular potential energy matrix $\mathbf{F}$ may be treated in a manner similar to that described in the TS calculation. Since it depends neither upon $\phi_{a}$ nor $\phi_{b}, \mathbf{F}\left(\phi_{a}, \phi_{b}, \phi_{c}\right)$ reduces to $\mathbf{F}\left(\phi_{c}\right)$. In the present calculation we have made use of the intramolecular potential function obtained by Schachtschneider and Snyder for a number of $n$-paraffins. ${ }^{25}$ Since we have chosen, however, the molecular parameters determined by Teare ${ }^{19}\left(r_{\mathrm{CC}}=1.5333 \AA, r_{\mathrm{CH}}=1.07 \AA, \angle \mathrm{CCC}=\right.$ $111.9^{\circ}$, and $\angle \mathrm{HCH}=107^{\circ}$ ), which are somewhat different from the tetrahedral geometry assumed by Schachtschneider and Snyder, we have modified their force constants to some extent. They obtained valence force constants based on the internal coordinates. We have transformed their $\mathbf{F}$ matrix into the one based on the local symmetry coordinates, ${ }^{14}$ and then, by examination of the Jacobian, changed the values of some of the diagonal elements. Diagonal elements of the $\mathrm{CH}_{2}$ symmetric stretching, $\mathrm{CH}_{2}$ bending, $\mathrm{CCC}$ bending, $\mathrm{CC}$ stretching, $\mathrm{CH}_{2}$ wagging, and $\mathrm{CH}_{2}$ twisting were changed, respectively, $-0.7 \%,-3.3 \%,-1.0 \%$, $-2.7 \%,-5.8 \%$, and $-8.3 \%$. The local symmetry coordinates employed here are not exactly the same as those used in the TS calculation, because it is necessary to make these coordinates orthogonal to the angle redundancy which, for nontetrahedral geometry, is not a mere sum of the six angles around a carbon atom. If the angles $\mathrm{CCC}, \mathrm{HCH}$, and $\mathrm{HCC}$ are represented by $\theta, \Phi$, and $\alpha_{i}$, then the redundant coordinate of the nontetrahedral $\mathrm{CH}_{2}$ group may be expressed $\mathrm{as}^{26}$

where

$$
a \Delta \theta+b \Delta \Phi+c\left(\Delta \alpha_{1}+\Delta \alpha_{2}+\Delta \alpha_{3}+\Delta \alpha_{4}\right),
$$

$$
\begin{aligned}
& a=\sin \theta \sin ^{2} \Phi(\cos \theta-1), \\
& b=\sin \Phi \sin ^{2} \theta(\cos \Phi-1), \\
& c=2 \sin \alpha \cos \alpha(1-\cos \theta)(1-\cos \Phi) .
\end{aligned}
$$

${ }_{25}$ J. H. Schachtschneider and R. G. Snyder, Spectrochim. Acta 19,117 (1963)

${ }^{26}$ See, for example, T. Onishi and T. Shimanouchi, Spectrochim, Acta 20, 325 (1964).

\section{RESULTS AND DISCUSSION}

\section{A. Effects of Cell Dimension and Setting Angle}

We have calculated the normal frequencies of the polyethylene crystal for two different cell dimensions, namely the dimensions at room temperature and those at liquid-nitrogen temperature, changing the setting angle from $38^{\circ}$ to $52^{\circ}$ in each case. The cell dimensions given by Swan ${ }^{27}$ were used: $a_{0}=7.414 \AA, b_{0}=4.942 \AA$ at room temperature, and $a_{0}=7.155 \AA, b_{0}=4.899 \AA$ at liquid-nitrogen temperature. In this calculation we assumed various $\mathrm{H} \cdots \mathrm{H}$ interatomic potential functions, as proposed by several authors. ${ }^{28,29}$ These are generally expressed as

$$
V_{\mathrm{HH}}=-A r^{-6}+B \exp (-C r),
$$

where $r$ is the $\mathrm{H} \cdots \mathrm{H}$ distance. We also used the potential given by Amdur et al. ${ }^{30}$ which is written as

$$
V_{\mathrm{HH}}=1.44 r^{-1.68} \mathrm{eV} \text {. }
$$

The potential given by (15) is for the range $2.09 \AA \leq r \leq 2.77 \AA$, but in our calculations we have extrapolated it to $r=2.95 \AA$. We evaluated the force constants $f_{i}$ by taking the second derivatives

\begin{tabular}{|c|c|c|c|c|}
\hline & de Boer & $\begin{array}{l}\text { Pitzer- } \\
\text { Catalano } \\
\text { de Boer }\end{array}$ & Bartell & $\begin{array}{l}\text { Amdur } \\
\text { et al. }\end{array}$ \\
\hline \multicolumn{5}{|c|}{ A. For unit cell parameters at $300^{\circ} \mathrm{K} ; \theta=48^{\circ}$} \\
\hline$f_{1}$ & 0.0043 & 0.0018 & 0.0020 & 0.0009 \\
\hline$f_{2}$ & 0.0048 & 0.0021 & 0.0023 & 0.0010 \\
\hline$f_{3}$ & 0.0159 & 0.0087 & 0.0128 & 0.0027 \\
\hline$f_{4}$ & 0.0180 & 0.0100 & 0.0150 & 0.0030 \\
\hline \multicolumn{5}{|c|}{ B. For unit cell parameters at $100^{\circ} \mathrm{K} ; \theta=48^{\circ}$} \\
\hline$f_{1}$ & 0.0053 & 0.0024 & 0.0020 & 0.0011 \\
\hline$f_{2}$ & 0.0071 & 0.0034 & 0.0023 & 0.0013 \\
\hline$f_{3}$ & 0.0251 & 0.0143 & 0.0128 & 0.0041 \\
\hline$f_{4}$ & 0.0205 & 0.0115 & 0.0150 & 0.0034 \\
\hline
\end{tabular}

$$
\left(\partial^{2} V_{\mathrm{HH}} / \partial r^{2}\right)_{r=q_{i}}
$$

of the potential curve. These force constants are listed in Table II for several potential functions. As

TABLE II. Intermolecular $H \cdot \cdots H$ force constants from various potential functions.

${ }^{27}$ P. R. Swan, J. Polymer Sci. 56, 403 (1962).

${ }^{28}$ Various potential functions are collected in the following paper: C. A. Coulson and C. W. Haigh, Tetrahedron 19, 527 (1963).

${ }_{29}$ R. A. Scott and H. A. Scheraga, J. Chem. Phys. 42, 2209 (1965).

${ }_{30}$ I. Amdur, M. S. Longmire, and E. A. Mason, J. Chem. Phys. $35,895(1961)$. 
representative examples, ${ }^{31}$ the results of the calculations for the potential functions of $\operatorname{deBoer}^{35}(A=0.0, B=$ $1.20 \times 10^{-10}, C=3.54 \times 10^{8} \mathrm{cgs}$ units), Pitzer, CatalanodeBoer $\left(A=3.42 \times 10^{-60}, B=1.20 \times 10^{-10}, C=3.54 \times 10^{8}\right)$

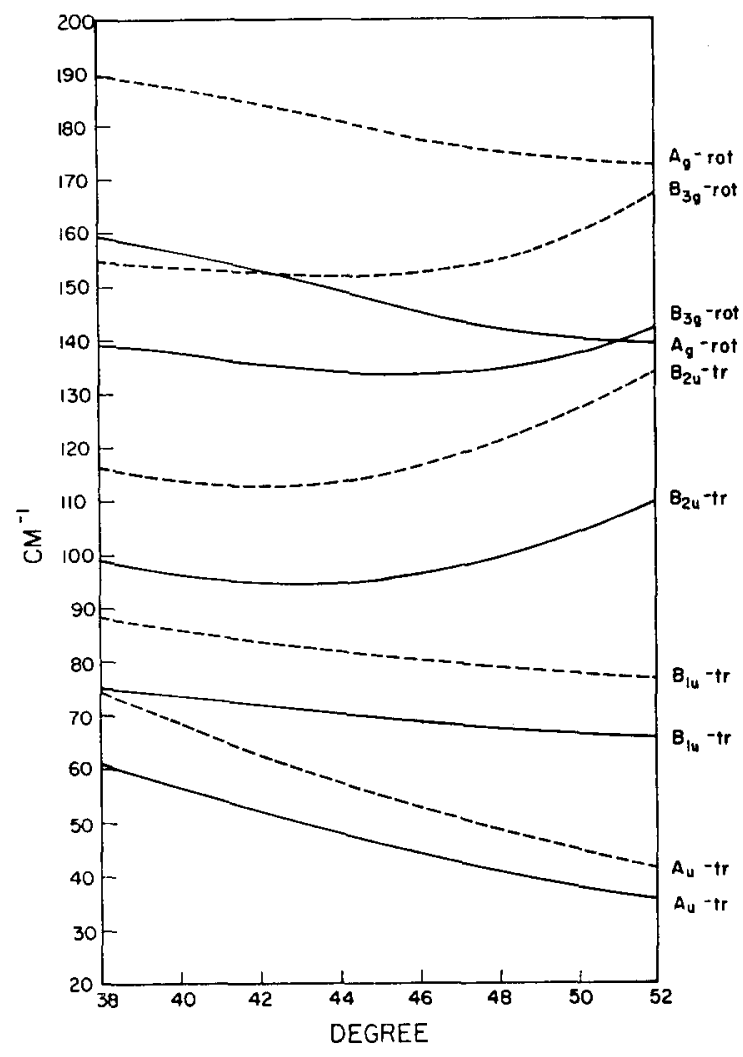

Frg. 2. Variation of low-frequency vibrations (tr is translation, rot is rotation) with setting angle; interactions calculated from the de Boer potential. -, $300^{\circ} \mathrm{K} ;----, 100^{\circ} \mathrm{K}$ cell parameters.

31 The four potential functions cited here seem to be most reliable from the viewpoint that (1) they give the calculated $B_{1 u}$ lattice frequency fairly close to the observed value, and (2) they can reasonably explain the temperature dependence of this band. Generally speaking, better results may be expected from a potential function which gives a slower dependence of $d^{2} V / d r^{2}$ on $r$. The Müller potentializ $\left(A=4.30 \times 10^{-60}, B=43.95 \times 10^{-10}\right.$, $\left.C=5.0 \times 10^{8}\right)$ has a fairly rapid change of $d^{2} V / d r^{2}$ with $r$, and hence gives much too large variations of the lattice frequencies with change in the setting angle and in the temperature. For example, the temperature dependence of the $B_{1 u}$ lattice frequency is more than $20 \mathrm{~cm}^{-1}$. The Scott-Scheraga potential ${ }^{29}(A=$ $3.14 \times 10^{-60}, B=6.37 \times 10^{-10}, C=4.54 \times 10^{8}$ ) gave negative eigenvalues for large setting angles, resulting from negative force constants for longer $\mathbf{H} \cdots \mathbf{H}$ distances. The Mason-Kreevoy potential $^{33}\left(A=6.21 \times 10^{-60}, B=2.58 \times 10^{-10}, C=3.07 \times 10^{8}\right)$, which was used by De Santis, Giglio, Liquori, and Ripamonti ${ }^{34}$ to calculate the conformational energy of polyethylene, would definitely give excessively high lattice frequencies since very large force constants are derived from this potential.

${ }^{32}$ A. Müller, Proc. Roy. Soc. (London) A154, 624 (1936); A178, 227 (1941).

${ }^{33}$ E. A. Mason and M. M. Kreevoy, J. Am. Chem. Soc. 77, 5808 (1955).

${ }^{34}$ P. De Santis, E. Giglio, A. M. Liquori, and A. Ripamonti, J. Polymer Sci. Pt. A 1, 1383 (1963).

${ }^{35} \mathrm{~J}$. deBoer, Physica 9, 363 (1942). Here we used only the repulsive part.

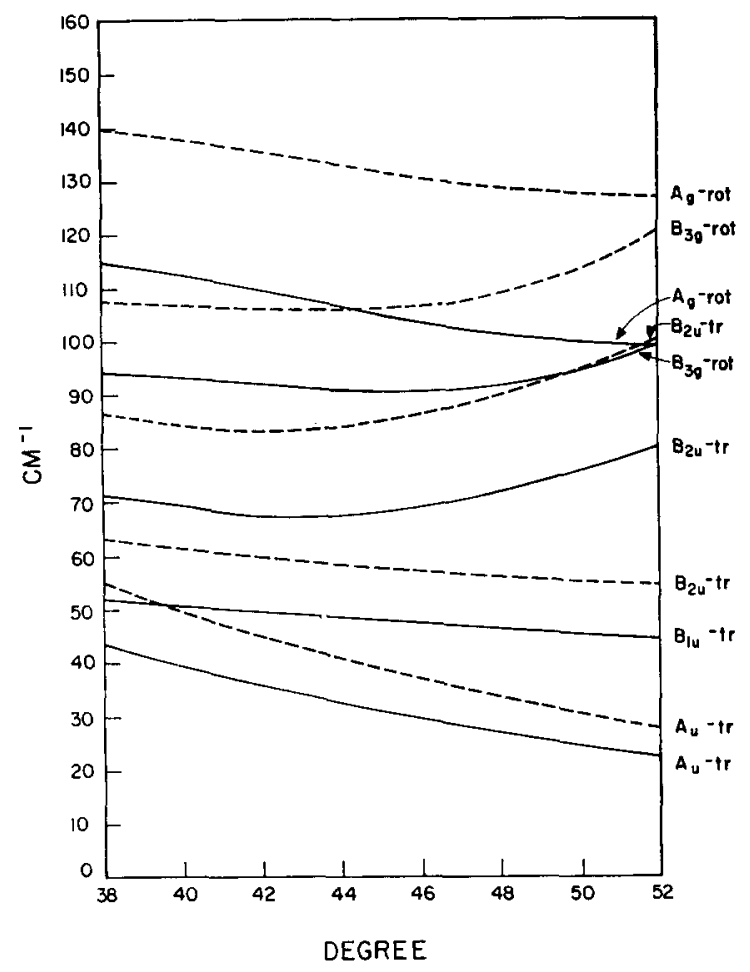

FIG. 3. Variation of low-frequency vibrations with setting angle; interactions calculated from the Pitzer, Catalano-de Boer potential.

(the attractive part is from the work of Pitzer and Catalano $\left.{ }^{36}\right)$, Bartell ${ }^{37}\left(A=3.42 \times 10^{-60}, B=4.58 \times 10^{-10}\right.$, $\left.C=4.08 \times 10^{8}\right)$, and Amdur et al. are shown in Figs. 2, 3,4 , and 5 . In these figures the dependence of the lattice frequencies on the cell dimensions and the setting angle is shown. As a consequence of the approximate nature of such potential functions, the results of these calculations are difficult to discuss very quantitatively. However, there is a strong indication that the contraction of the cell dimensions with decreasing temperature has a significant influence in increasing the lattice vibration frequencies. The lattice frequencies also vary with the setting angle. These results therefore make it quite reasonable to associate the upward shift of the $B_{1 u}$ translational lattice frequency at low temperatures with the change of normal frequency and normal coordinate which accompanies the cell contraction. ${ }^{2}$

In each case the shift is calculated to be $10 \mathrm{~cm}^{-1}$ or more, even though the observed shift is only $6.5 \mathrm{~cm}^{-1} \cdot{ }^{10}$ This discrepancy may be due to anharmonic effects. Because of the symmetry of the translational mode, the cubic term is absent when the intermolecular potential function (14) is summed over the unit cell.

${ }^{36}$ K. S. Pitzer and E. Catalano, J. Am. Chem. Soc. 78, 4844 (1956).

${ }^{37}$ L. S. Bartell, J. Chem. Phys. 32, 827 (1960). 
The next-higher allowed term is the quartic term. A simple calculation shows that the coefficient of the quartic term is of the same sign as that of the quadratic term. For example, consider the chains replaced by points at the corners and center of the polyethylene unit cell. For a vibration of small amplitude parallel to the $a$ axis ( $x$ direction), the potential in which the center chain moves is given by

$$
\begin{aligned}
V(x) & =2\left\{-A(R-x \cos \omega)^{-6}+B \exp [-C(R-x \cos \omega)]-A(R+x \cos \omega)^{-6}+B \exp [-C(R+x \cos \omega)]\right\} \\
& =2\left(-A R^{-6}\left[(1-y)^{-6}+(1+y)^{-6}\right]+B\{\exp [-C R(1-y)]+\exp [-C R(1+y)]\}\right) .
\end{aligned}
$$

In this equation, $R$ is the distance between the center chain and one at the corner, $\omega$ is the angle between this line and the $x$ axis, and $y=x \cos \omega / R$. For small oscillations ( $x$ not larger than $\sim 0.1 \AA$ ) $y$ is small compared to 1 , and the first expression, in braces, can be suitably approximated. Expansion of the exponential then gives

$$
\begin{aligned}
V(x)=2\left(-A R^{-6}(2+\right. & \left.30 y^{2}+30 y^{4}+\cdots\right)+B\left\{\left[2-2 C R+(C R)^{2}-\frac{1}{3}(C R)^{3}+\frac{1}{12}(C R)^{4}-\frac{1}{60}(C R)^{5}+\cdots\right]\right. \\
& \left.\left.+\left[(C R)^{2}-(C R)^{3}+\frac{1}{2}(C R)^{4}-\frac{1}{6}(C R)^{5}+\cdots\right] y^{2}+\left[\frac{1}{12}(C R)^{4}-\frac{1}{12}(C R)^{5}+\cdots\right] y^{4}+\cdots\right\}\right) .
\end{aligned}
$$

From (17) it is possible to see that the coefficients of $y^{2}$ and $y^{4}$ have the same sign in the first term, and also that this is maintained in the second term, particularly as higher powers of $(C R)$ begin to dominate. With a Lennard-Jones potential this is more directly seen because the expansion of the inverse 12 th power term can be terminated at $y^{4}$, as was done for the first term in (16). In the translational mode of the actual chain all of the $\mathrm{H} \cdot . \mathrm{H}$ distances between a corner chain and a center chain increase and decrease together (see Fig. 1), so that it seems reasonable that the above argument will also apply to this case. Thus, the departure from a harmonic potential in this constant-volume case is such that the separation between vibrational energy levels increases as the quantum number increases. As a result, diminishing temperature, and consequent depopulation of higher energy levels, will lead to a downward shift in frequency, not an upward shift as previously suggested. ${ }^{10}$ The combination of this effect with the upward shift arising from the closer approach of the chains at low temperature could account for the fact that the observed upward shift is less than that calculated from the atom-atom interaction. A more exact prediction would require detailed knowledge of the anharmonicity.

The frequency distribution for the polyethylene crystal was calculated recently and from this the specific heat was derived. ${ }^{9}$ This calculation is based on the cell dimensions at room temperature. The present results indicate that it might be worth while taking into account in this calculation the contraction of the cell, particularly in treating the specific heat at very low temperatures.

The splitting of the infrared-active internal $\mathrm{CH}_{2}$ vibrations is also dependent on the cell dimensions and on the setting angle. The results of calculations of such splittings are summarized in Table III. The variation with the setting angle is nearly linear in each case. It can be seen that while splittings of the right order of magnitude are predicted for the $\mathrm{CH}_{2}$ rocking mode this is not true for the $\mathrm{CH}_{2}$ bending mode. The reason for this is not clear. A calculation done with a $\mathrm{C}-\mathrm{H}$ bond length of $1.093 \AA$ gave no significant improvement.

\section{B. Intermolecular Hydrogen-Hydrogen Force Constants}

It was felt difficult, from the calculation based on assumed potential functions, to determine the best setting angle and simultaneously to get a good agreement between the calculated and observed values of the $B_{1 u}$ translational lattice frequency and the splittings

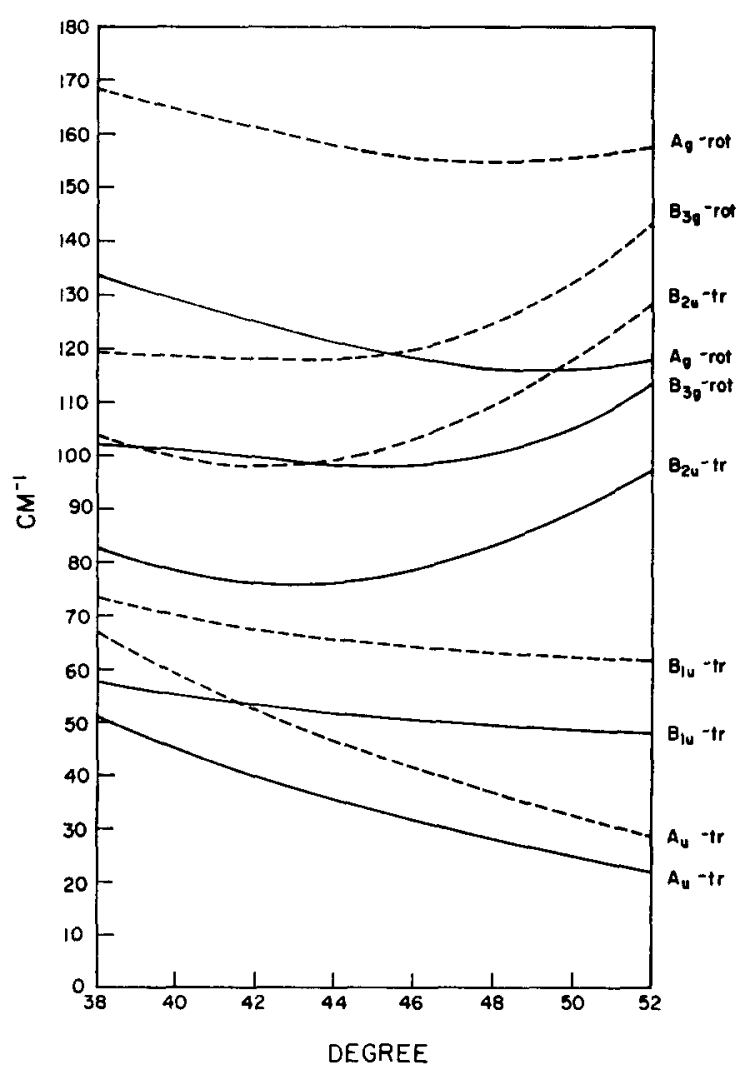

FIG. 4. Variation of low-frequency vibrations with setting angle; interaction calculated from Bartell potential. 
TABLE III. Dependence of the calculated splittings of the $\mathrm{CH}_{2}$ vibration frequencies on temperature and setting angles.

\begin{tabular}{|c|c|c|c|c|c|c|c|c|c|}
\hline \multirow{3}{*}{ Potential function } & \multirow{3}{*}{ Tempa } & \multicolumn{8}{|c|}{ Splitting, $\bar{\nu}\left(B_{1 u}\right)-\bar{\nu}\left(B_{2 u}\right)$} \\
\hline & & \multicolumn{2}{|c|}{$\mathrm{CH}_{2}$ bend } & \multicolumn{2}{|c|}{$\mathrm{CH}_{2}$ rock } & \multicolumn{2}{|c|}{$\mathrm{CH}_{2}$ sym stretch } & \multicolumn{2}{|c|}{$\mathrm{CH}_{2}$ antisym stretch } \\
\hline & & $\theta=38^{\circ}$ & $\theta=52^{\circ}$ & $\theta=38^{\circ}$ & $\theta=52^{\circ}$ & $\theta=38^{\circ}$ & $\theta=52^{\circ}$ & $\theta=38^{\circ}$ & $\theta=52^{\circ}$ \\
\hline de Boer & $\begin{array}{l}100^{\circ} \mathrm{K} \\
300^{\circ} \mathrm{K}\end{array}$ & $\begin{array}{r}12.0 \\
9.3\end{array}$ & $\begin{array}{l}3.1 \\
3.5\end{array}$ & $\begin{array}{l}17.8 \\
12.9\end{array}$ & $\begin{array}{l}35.2 \\
24.6\end{array}$ & $\begin{array}{l}-6.4 \\
-4.6\end{array}$ & $\begin{array}{r}+8.9 \\
+5.4\end{array}$ & $\begin{array}{l}+5.2 \\
+3.5\end{array}$ & $\begin{array}{l}-9.9 \\
-6.4\end{array}$ \\
\hline Pitzer, Catalano-de Boer & $\begin{array}{l}100^{\circ} \mathrm{K} \\
300^{\circ} \mathrm{K}\end{array}$ & $\begin{array}{l}6.2 \\
4.5\end{array}$ & $\begin{array}{l}0.2 \\
0.5\end{array}$ & $\begin{array}{l}7.0 \\
5.2\end{array}$ & $\begin{array}{l}18.7 \\
13.2\end{array}$ & $\begin{array}{l}-3.6 \\
-2.5\end{array}$ & $\begin{array}{l}+5.4 \\
+3.3\end{array}$ & $\begin{array}{l}+3.0 \\
+2.0\end{array}$ & $\begin{array}{l}-5.8 \\
-3.7\end{array}$ \\
\hline Bartell & $\begin{array}{l}100^{\circ} \mathrm{K} \\
300^{\circ} \mathrm{K}\end{array}$ & $\begin{array}{l}8.6 \\
5.9\end{array}$ & $\begin{array}{l}-2.8 \\
-1.1\end{array}$ & $\begin{array}{l}6.6 \\
4.6\end{array}$ & $\begin{array}{l}31.2 \\
19.0\end{array}$ & $\begin{array}{l}-5.7 \\
-3.6\end{array}$ & $\begin{array}{l}+9.8 \\
+5.4\end{array}$ & $\begin{array}{l}+4.8 \\
+2.9\end{array}$ & $\begin{array}{r}-10.2 \\
-5.7\end{array}$ \\
\hline Amdur et al. & $\begin{array}{l}100^{\circ} \mathrm{K} \\
300^{\circ} \mathrm{K}\end{array}$ & $\begin{array}{l}2.2 \\
1.8\end{array}$ & $\begin{array}{l}1.0 \\
1.0\end{array}$ & $\begin{array}{l}3.5 \\
2.9\end{array}$ & $\begin{array}{l}6.2 \\
4.4\end{array}$ & $\begin{array}{l}-1.0 \\
-0.7\end{array}$ & $\begin{array}{l}+1.4 \\
+0.8\end{array}$ & $\begin{array}{l}+1.2 \\
+0.6\end{array}$ & $\begin{array}{l}-1.6 \\
-1.0\end{array}$ \\
\hline
\end{tabular}

a Cell dimensions at these temperatures taken from Swan.27

of the $\mathrm{CH}_{2}$ bending and rocking frequencies. As a next step we decided therefore to calculate the "best" set of force constants for the most likely value of the setting angle. Although there has been some question concerning the value of the setting angle obtained from $x$-ray diffraction studies, the most probable value seems to be around $48^{\circ} .19,88$ The experimental spectroscopic data used to determine the force constants at room and liquid nitrogen temperatures are given in Table IV, together with the calculated values of the $B_{1 u}$ lattice frequency and the splittings. We have

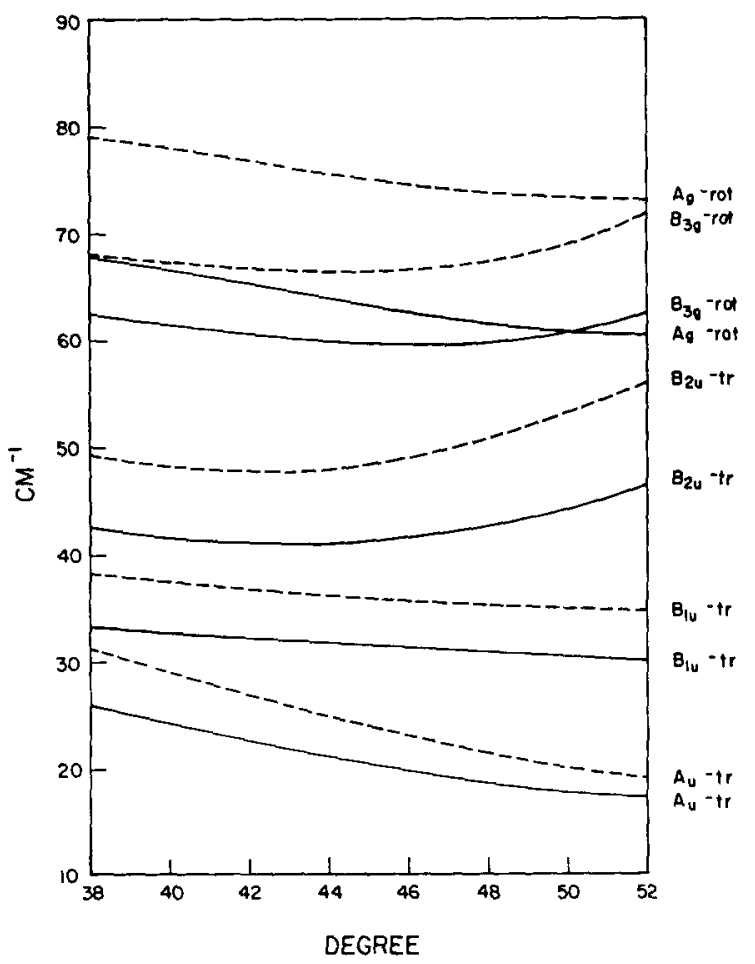

FIG. 5. Variation of low-frequency vibrations with setting angle; interactions calculated from Amdur potential.

${ }^{88}$ N. Kasai and M. Kakudo, Mezhdunar. Simpozium p. Makromdekul. Khim. Prague 1965, 491 (to be published). obtained two sets of force constants, both of which are listed in Table V. Set I gives a better calculated $B_{1 u}$ lattice frequency, and Set II is superior in explaining the splittings of the $\mathrm{CH}_{2}$ bending and rocking vibrations. Set II is more useful when the problem of the splitting in mixed crystals of normal and deuterated polyethylenes is treated ${ }^{39}$ The agreement between the calculated and observed frequencies would probably be improved if longer interatomic interactions were taken into account, and possibly also if $\mathrm{C} \cdots \mathrm{H}$ interactions were included. Of course, if anharmonicity is significant, then these harmonic force constants would have to be modified.

\section{Dispersion Curves}

As an extension of the study described in the preceding section, we calculated the dispersion curves of both $-\left(\mathrm{CH}_{2}\right)_{n}-$ and $-\left(\mathrm{CD}_{2}\right)_{n}$ - chains under the conditions $\phi_{c}=\phi_{b}=0$. Normal frequencies were computed at an interval of $1^{\circ}$ between $\delta_{c}=0^{\circ}$ and $20^{\circ}$, at an interval of $10^{\circ}$ between $20^{\circ}$ and $160^{\circ}$, and again at an interval of $1^{\circ}$ between $160^{\circ}$ and $180^{\circ}$. As a result, detailed information was obtained at both ends of the dispersion curves, which is particularly necessary in order to interpret the neutron scattering peaks. The results of the calculations for the cell dimensions at liquidnitrogen temperature using Set I force constants are shown in Figs. 6 and 7. In these figures the full and broken lines refer, respectively, to the $\nu^{a}$ and $\nu^{b}$ branches. ${ }^{14}$ The $\nu^{a}$ branch is related to the spectroscopically active modes which are polarized along the $a$ axis, and the $\nu^{b}$ branch to the modes having $b$-axis polarization. On deuteration the dispersion curves for modes other than $\nu_{5}$ and $\nu_{9}$ change significantly from those of the $-\left(\mathrm{CH}_{2}\right)_{-n}$ chain. It might be worthwhile to describe the vibrational modes of each of the curves of the deuterated polyethylene. A detailed analysis of the vibrational modes of normal polyethylene was presented previously. ${ }^{40}$ Here we repeat it briefly to

${ }^{39}$ M. Tasumi and S. Krimm (to be published).

${ }^{40} \mathrm{M}$. Tasumi, T. Shimanouchi, and T. Miyazawa, J. Mol. Spectry. 9, 261 (1962). 
TABLE IV. Observed and calculated values of the $B_{1 u}$ lattice frequency and the splitting of $\mathrm{CH}_{2}$ bending and rocking modes $\left(\mathrm{cm}^{-1}\right)$.

\begin{tabular}{|c|c|c|c|c|c|c|}
\hline & \multicolumn{3}{|c|}{ Room temperature } & \multicolumn{3}{|c|}{ Liquid-nitrogen temperature } \\
\hline & Obs & Calc I & Calc II & Obs & Calc I & Calc II \\
\hline$B_{1 u}$ lattice frequency & 72.5 & 71.1 & 78.5 & 79.0 & 80.6 & 85.9 \\
\hline $\mathrm{CH}_{2}$ bending splitting & 10.0 & 9.3 & 9.9 & $13.0^{\mathrm{b}}$ & 12.4 & 13.0 \\
\hline $\mathrm{CH}_{2}$ rocking splitting & 11.0 & 8.5 & 10.5 & $12.9^{c}$ & 11.9 & 13.3 \\
\hline
\end{tabular}

Calc I and II were obtained from Set I and Set II force constants.

b This value was taken from Ref. 22 . c This value was taken from G. W. King, R. M. Hainer, and H. O. McMahon, J. Appl. Phys. 20, 559 (1949). make easier the comparison of modes of normal and deuterated polyethylenes.

\section{Normal Polyethylene}

The branches $\nu_{6}$ and $\nu_{1}$ are, respectively, the $\mathrm{CH}_{2}$ antisymmetric and symmetric stretching vibrations. $\nu_{2}$ is the $\mathrm{CH}_{2}$ bending vibration branch. $\nu_{3}$ is mainly the $\mathrm{CH}_{2}$ wagging mode, the $\mathrm{CC}$ stretching mode being mixed in when $\delta_{c}$ is close to $\pi . \nu_{4}$ is the skeletal stretching vibration. $\nu_{4}(0)$ is the symmetric (Raman active) mode in which the $\mathrm{CC}$ stretching and $\mathrm{CCC}$ bending are coupled out of phase, and $\nu_{4}$ at about $\delta_{c}=60^{\circ}$ is almost purely $\mathrm{CC}$ stretching. The $\mathrm{CH}_{2}$ wagging mode is mixed in at $\delta_{c}=\pi$. In $\nu_{7}$ and $\nu_{8}$ both the $\mathrm{CH}_{2}$ rocking and twisting vibrations are mixed. $\nu_{7}(0)$ and $\nu_{8}(0)$ are, respectively, the pure $\mathrm{CH}_{2}$ rocking and twisting. As $\delta_{c}$ increases, the twisting component increases in $\nu_{7}$, and the rocking in $\nu_{8}, \nu_{7}(\pi)$ and $\nu_{8}(\pi)$ are the pure $\mathrm{CH}_{2}$ twisting and rocking, respectively.

\section{Deuterated Polyethylene}

The branches $\nu_{6}$ and $\nu_{1}$ are, respectively, the $\mathrm{CD}_{2}$ antisymmetric and symmetric stretching vibrations. The branches $\nu_{4}, \nu_{2}, \nu_{3}$ are interrelated. $\nu_{4}(0)$ is the symmetric skeletal stretching vibration, but with increase of $\delta_{c}$ the $\mathrm{CD}_{2}$ wagging component increases in $\nu_{4}$ until $\delta_{c}=60^{\circ}$. In the region $\delta_{c}>60^{\circ}$ the wagging com- ponent decreases, and $\nu_{4}(\pi)$ is the antisymmetric skeletal stretching vibration. $\nu_{2}(0)$ is the symmetric (Raman active) $\mathrm{CD}_{2}$ bending vibration slightly coupled with the $\mathrm{CD}_{2}$ rocking. The wagging component becomes mixed in $\nu_{2}$ also until $\delta_{c}=60^{\circ}$ and then decreases. $\nu_{2}(\pi)$ is the antisymmetric (infrared-active) $\mathrm{CD}_{2}$ bending vibration. $\nu_{3}(0)$ is the infrared active $\mathrm{CD}_{2}$ wagging vibration. The $\mathrm{CC}$ stretching component increases in $\nu_{3}$ as $\delta_{c}$ increases, and $\nu_{3}\left(60^{\circ}\right)$ is an almost pure CC stretching vibration. For $\delta_{c}>60^{\circ}$ the wagging component increases again, and $\nu_{3}(\pi)$ is the Ramanactive $\mathrm{CD}_{2}$ wagging vibration. The branches $\nu_{7}$ and $\nu_{8}$ are an interrelated pair. $\nu_{7}(0)$ is the Raman-active $\mathrm{CD}_{2}$ rocking slightly coupled with the $\mathrm{CD}_{2}$ bending. As $\delta_{c}$ increases the $\mathrm{CD}_{2}$ twisting component increases, and $\nu_{7}(\pi)$ is the Raman-active $\mathrm{CD}_{2}$ twisting vibration. $\nu_{8}(0)$ is the $\mathrm{CD}_{2}$ twisting vibration, and the rocking component becomes mixed as $\delta_{e}$ increases. $\nu_{8}(\pi)$ is the infrared-active $\mathrm{CD}_{2}$ rocking vibration.

It was found that the intramolecular force constants used here failed to give better agreement between the calculated and observed frequencies of the $-\left(\mathrm{CD}_{2}\right){ }_{n}$ chain than did the force constants used in the TS calculation, although better agreement was obtained for the $-\left(\mathrm{CH}_{2}\right){ }_{n}$ frequencies. This may be due to the fact that anharmonicities are important for hydrogen vibrations, and therefore a harmonic force field is an approximation. Since these anharmonicities are different for deuterium vibrations, it is not surprising

TABLE V. H...H distances (in angstroms) and force constants (in millidynes per angstrom) at $\theta=48^{\circ}$.

\begin{tabular}{|c|c|c|c|c|c|c|c|c|c|}
\hline \multicolumn{5}{|c|}{ Room temperature } & \multicolumn{5}{|c|}{ Liquid-nitrogen temperature } \\
\hline & & & Set I & Set II & & & & Set I & Set II \\
\hline$q_{1}$ & 2.954 & $f_{1}$ & 0.0034 & 0.0035 & $q_{1}$ & 2.894 & $f_{1}$ & 0.0048 & 0.0050 \\
\hline$q_{2}$ & 2.925 & $f_{2}$ & 0.0109 & 0.0144 & $q_{2}$ & 2.816 & $f_{2}$ & 0.0125 & 0.0150 \\
\hline$q_{3}$ & 2.586 & $f_{3}$ & 0.0130 & 0.0200 & $q_{3}$ & 2.457 & $f_{3}$ & 0.0142 & 0.0188 \\
\hline$q_{4}$ & 2.551 & $f_{4}^{\mathbf{a}}$ & 0.0180 & 0.0180 & $q_{4}$ & 2.514 & $f_{4}{ }^{a}$ & 0.0205 & 0.0205 \\
\hline
\end{tabular}

a This value was determined from the de Boer potential and used in the calculation since it cannot be determined from the lattice freguencies and is only involved in second order in the splitting of the infrared-active internal modes. 


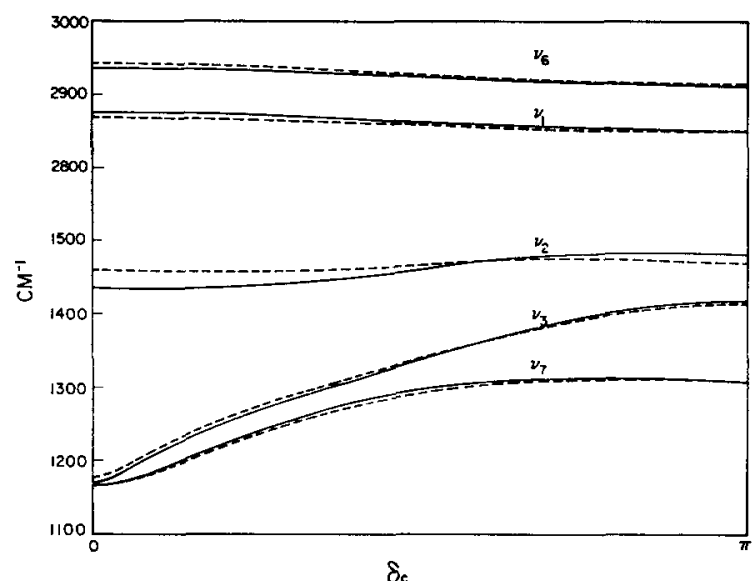

(a)

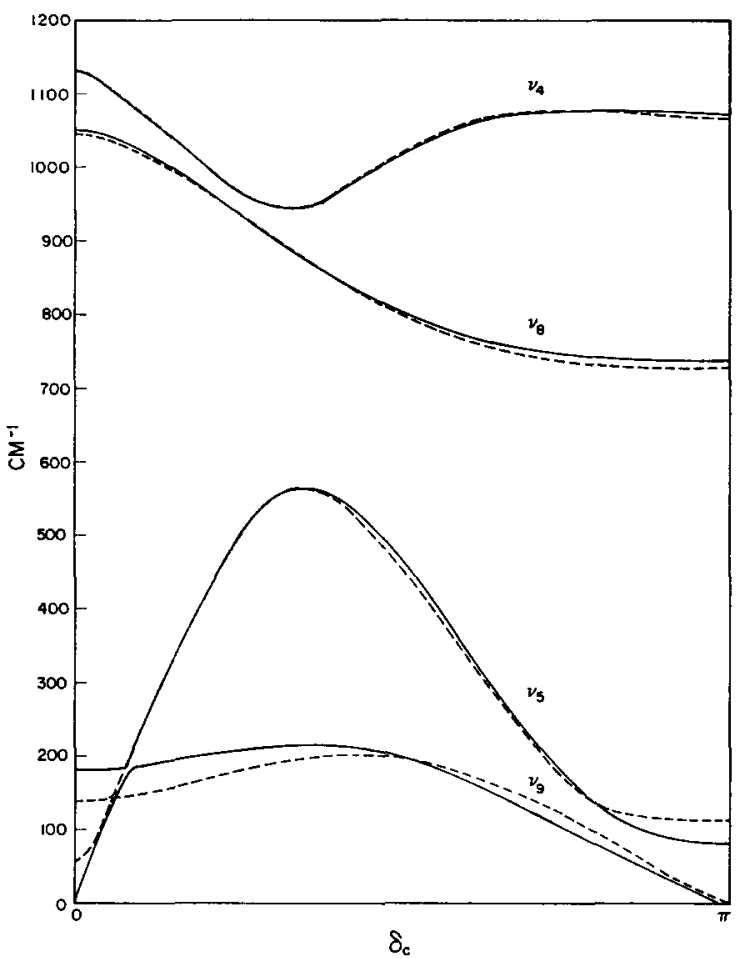

(b)

Fig. 6. Dispersion curves for $-\left(\mathrm{CH}_{2}\right)_{-n}$, for $100^{\circ} \mathrm{K}$ cell parameters. - $-\nu^{a}$ branch;,$---- \nu^{b}$ branch.

that the harmonic constants fitted to the hydrogen frequencies are not transferable in detail to the deuterated molecule. Nevertheless, the general features of the dispersion curves calculated here for the $-\left(\mathrm{CD}_{2}\right){ }_{n}$ chain are probably not significantly in error.

The temperature dependence of the low-frequency vibrations of both the $-\left(\mathrm{CH}_{2}\right)-_{n}$ and $-\left(\mathrm{CD}_{2}\right)-_{n}$ chains are summarized in Table VI. This may be of some value in interpreting the results of neutron-scattering experiments. In Table VI the results of the calculation ing the Set $I$ force constants are given. $\nu_{5}{ }^{a}(0)$ and $\nu_{5}^{\circ}(0)$ are, respectively, the $A_{v}$ and $B_{3 q}$ rotational

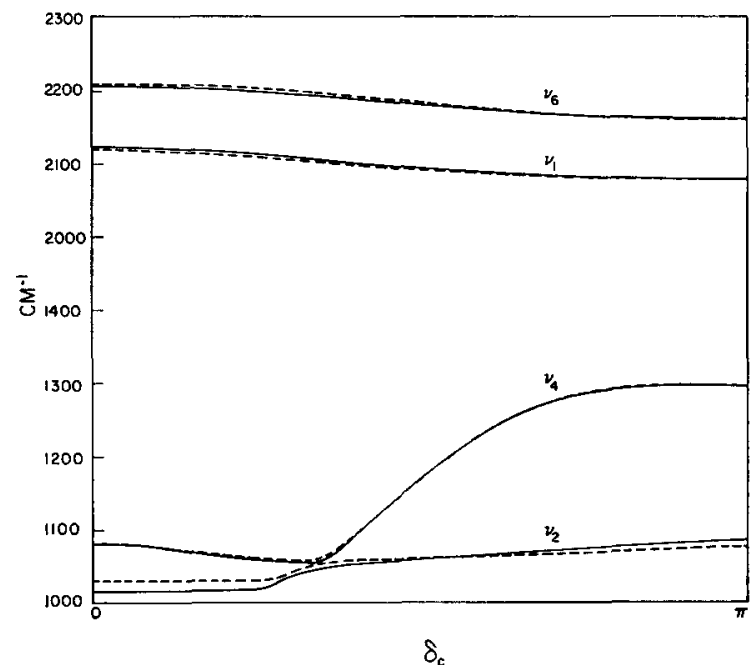

(a)

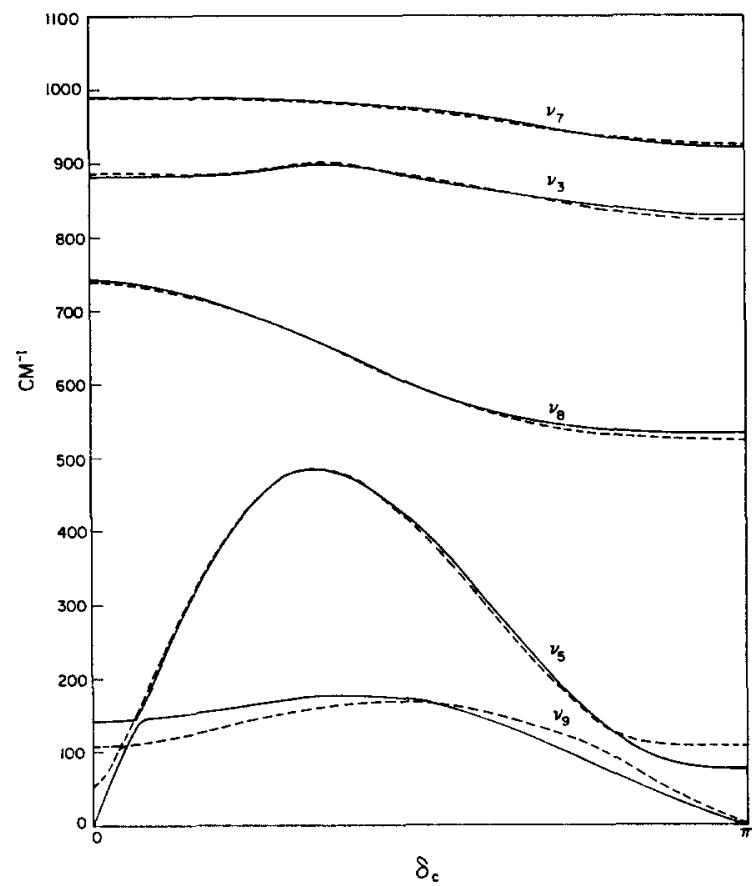

(b)

Fig. 7. Dispersion curves for $-\left(\mathrm{CD}_{2}\right)-{ }_{n}$, for $100^{\circ} \mathrm{K}$ cell parameters. - - $\nu^{a}$ branch;,$---- \nu^{b}$ branch.

TABLE VI. Temperature dependence of low frequencies of the $-\left(\mathrm{CH}_{2}\right)_{n}$ and $-\left(\mathrm{CD}_{2}\right)_{n}$ chains $\left(\mathrm{cm}^{-1}\right)$.

\begin{tabular}{lcccc}
\hline \multicolumn{1}{c}{ Mode } & \multicolumn{2}{c}{$\begin{array}{c}\text { Room } \\
\text { temperature }\end{array}$} & \multicolumn{2}{c}{$\begin{array}{c}\text { Liquid-nitrogen } \\
\text { temperature }\end{array}$} \\
\hline & $-\left(\mathrm{CH}_{2}\right)-_{n}$ & $-\left(\mathrm{CD}_{2}\right)-_{n}$ & $-\left(\mathrm{CH}_{2}\right)-n$ & $-\left(\mathrm{CD}_{2}\right)-_{n}$ \\
$\nu_{5}{ }^{a}(0)$ & 171.5 & 134.8 & 181.7 & 142.9 \\
$\nu_{5}{ }^{b}(0)$ & 121.2 & 95.0 & 138.8 & 108.9 \\
$\nu_{9^{b}}(0)$ & 50.7 & 47.5 & 57.5 & 53.9 \\
$\nu_{5}{ }^{a}(\pi)$ & 71.1 & 66.6 & 80.6 & 75.6 \\
$\nu_{5}^{b}(\pi)$ & 108.6 & 102.0 & 113.3 & 106.5 \\
$\nu_{5}{ }^{a}$ cutoff & $\sim 563$ & $\sim 483$ & $\sim 564$ & $\sim 484$ \\
$\nu_{5}^{b}$ cutoff & $\sim 563$ & $\sim 483$ & $\sim 565$ & $\sim 485$ \\
$\nu_{9}{ }^{a}$ cutoff & $\sim 208$ & $\sim 171$ & $\sim 215$ & $\sim 177$ \\
$\nu_{9}{ }^{b}$ cutoff & $\sim 196$ & $\sim 163$ & $\sim 204$ & $\sim 169$ \\
\hline \hline
\end{tabular}


lattice vibrations. $\nu_{9}^{b}(0)$ is the $A_{u}$ translational lattice vibration along the chain direction, and $\nu_{5}{ }^{a}(\pi)$ and $\nu_{5}^{b}(\pi)$ are, respectively, the $B_{1 u}$ and $B_{2 u}$ translational lattice vibrations. It might be noted that Raman spectra of polyethylene have not yet been obtained in the region of the $\nu_{5}^{a}(0)$ and $\nu_{5}^{b}(0)$ modes. Such data would be of help in verifying the correctness of the intermolecular force field.

\section{ACKNOWLEDGMENT}

This research was supported by a grant from the National Science Foundation.

\title{
Series Solutions and Rotationless States of the Vibrating Hydrogen Molecule Ion*
}

\author{
DONALD K. HaRriss \\ Department of Chemistry, University of Minnesota, Duluth, Duluth, Minnesota
}

(Received 28 July 1966)

\begin{abstract}
The generalized series solution methods for nonseparable Hamiltonians proposed by Frost are investigated. It is shown that for the general case a solution is not possible without explicit evaluation of a minimum number of integrals. The $\mathbf{B}$ matrix method is shown to be equivalent to the use of the integral relations, $J(f)$, developed in Hirschfelder's derivation of quantum-mechanical hypervirial theorems. The B matrix method is applied to vibrating $\mathrm{H}_{2}^{+}, \mathrm{HD}^{+}$, and $\mathrm{D}_{2}{ }^{+}$.
\end{abstract}

\section{INTRODUCTION}

$\mathbf{T}$ HE application of series solution methods to the solution of nonseparable multidimensional Schrödinger equations has been developed by Pekeris, ${ }^{1}$ and by Frost and co-workers. ${ }^{2-4}$ With the exceptions of hydrogenlike atoms ${ }^{2}$ and three-particle systems using perimetric coordinates, ${ }^{1,3}$ it has not been found possible with these methods to obtain a solution without the explicit evaluation of at least a minimum number of integrals.

The purpose of this work is to consider the question of whether, in general, a series solution is possible without evaluation of integrals. The methods of solution proposed by Frost ${ }^{2}$ are discussed and the relationship between the B matrix method ${ }^{2,4}$ and the $J(f)$ integral relations developed by Hirschfelder ${ }^{5-7}$ is shown.

For illustration, the $B$-matrix method is applied to the vibrating hydrogen molecule-ion. Although the vibrational eigenvalues of this system are well known, ${ }^{8}$

* Acknowledgment is made to the donors of the Petroleum Research Fund, administered by the American Chemical Society, for partial support of this research. Partial support was also provided by the Graduate School of the University of Minnesota.

${ }^{1}$ C. L. Pekeris, Phys. Rev. 112, 1649 (1958); 115, 1216 (1959); 126, 143, 1470 (1962); 127, 509 (1962); C. L. Pekeris, B. Schiff, and H. Lifson, ibid. 126, 1057 (1962).

2 A. A. Frost, J. Chem. Phys. 41, 478 (1964).

${ }^{3}$ A. A. Frost, M. Inokuti, and J. P. Lowe, J. Chem. Phys. 41, 482 (1964).

${ }^{4}$ A. A. Frost, D. K. Harriss, and J. D. Scargle, J. Chem. Phys. 41, 489 (1964).

5 J. O. Hirschfelder, J. Chem. Phys. 33, 1462 (1960).

S. T. Epstein and J. O. Hirschfelder, Phys. Rev. 123, 1495 (1961).

${ }^{7} \mathrm{~J}$. O. Hirschfelder and C. A. Coulson, J. Chem. Phys. 36, 941 (1962)

${ }^{8}$ H. Wind, J. Chem. Phys. 43, 2956 (1965). it has not proven amenable to treatment by series solution as a three-particle system in perimetric coordinates. $^{3}$

\section{REVIEW OF THE SERIES SOLUTION METHOD}

Using the notation of Frost, ${ }^{2}$ the series solution method can be outlined in the following manner. An attempt is made to solve the Schrödinger equation

$$
\mathfrak{H C} \Psi=E \Psi
$$

using the infinite series

$$
\Psi=\sum_{j} \phi_{j} c_{j}
$$

where the basis functions $\phi_{j}$ are a complete, linearly independent set satisfying the appropriate boundary conditions. Then

$$
\sum_{j}\left(\mathfrak{H} \phi_{j}-E \phi_{j}\right) c_{j}=0 .
$$

If an attempt is made to expand $3 C \phi_{j}$ in (3) as a linear combination of the $\phi_{i}$, it is found that the expansion is not possible if the $\phi_{j}$ are expressed in the form of exponential power series of the interparticle coordinates since the reciprocals of these coordinates occur in the Hamiltonian. This difficulty can be surmounted by multiplication of (3) by a suitable function, $g$, followed by expansions as

$$
g \mathfrak{H} \phi_{j}=\sum_{i} \phi_{i} H_{i j}
$$

and

$$
g \phi_{j}=\sum_{i} \phi_{i} S_{i j}
$$

